\title{
Analysis of Native-Like Ions using \\ Structures for Lossless Ion Manipulations
}

Samuel J. Allen, Rachel M. Eaton, Matthew F. Bush*

\section{SUPPORTING INFORMATION}

Contribution from the:

University of Washington, Department of Chemistry, Box 351700

Seattle, WA 98195-1700

*Address correspondence to mattbush@uw.edu

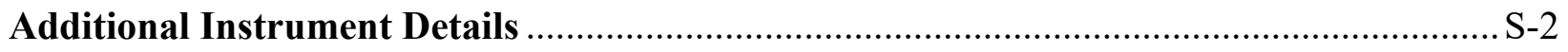

Note on use of drift times versus corrected drift times................................................... S-3

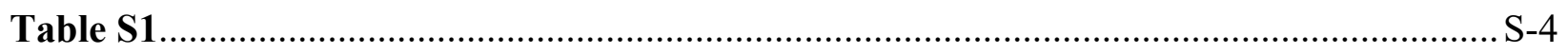

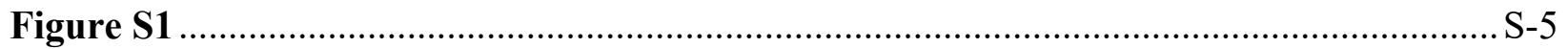

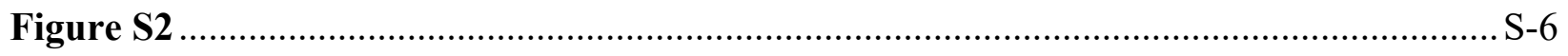

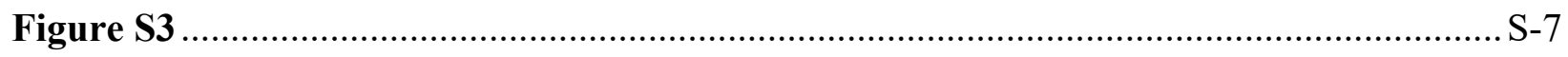

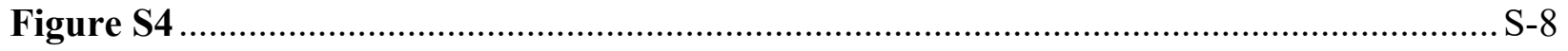

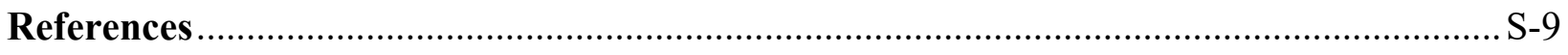




\section{Additional Instrument Details}

Ion Funnel Trap. The ion funnel trap consists of 190 ring electrode boards that have $0.70 \mathrm{~mm}$ thicknesses and $1.71 \mathrm{~mm}$ electrode center-to-center spacing. The electrode inner diameters range from $25.4 \mathrm{~mm}$ at the entrance to $2.3 \mathrm{~mm}$ at the exit of the funnel. Three gate electrodes were constructed by attaching $95 \%$ transmission nickel mesh over $25.4 \mathrm{~mm}$ inner diameter ring electrode boards. The trapping region, designated as the distance from the entrance gate to the trap and exit gates, is $20.5 \mathrm{~mm}$. Ions pass from the ion funnel trap through a $1.02 \mathrm{~mm}$ thick copper electrode with a $1.85 \mathrm{~mm}$ inner diameter aperture into a second vacuum chamber that contains a rectangular ion funnel, the SLIM boards, and a circular ion funnel.

Rectangular Ion Funnel. The rectangular ion funnel ${ }^{1}$ consists of 142 printed circuit boards that have thicknesses of $0.61 \mathrm{~mm}$ and electrode center-to-center spacing of $1.23 \mathrm{~mm}$. The inner diameters of the electrodes narrow from $25.4 \mathrm{~mm}$ at the entrance to $5.36 \mathrm{~mm}$ at the exit. Similar to the ion funnel trap, resistors, capacitors, and spring-loaded connectors were soldered directly to the boards. Ions exiting the rectangular ion funnel were passed directly into the SLIM board region.

Circular Ion Funnel. The circular ion funnel consists of 114 ring electrode boards that have thicknesses of $0.66 \mathrm{~mm}$ and electrode center-to-center spacing of $1.28 \mathrm{~mm}$. The inner diameters of the ring electrodes range from $25.4 \mathrm{~mm}$ at the entrance to $2.50 \mathrm{~mm}$ at the exit. Similar to the ion funnel trap and rectangular ion funnel, resistors, capacitors, and spring-loaded connectors were soldered directly onto the boards. A second conductance limit, $0.762 \mathrm{~mm}$ thick aluminum with an inner diameter of $1.02 \mathrm{~mm}$, is positioned between the terminus of the circular ion funnel and the stacked-ring ion guide of the original Waters Q-Tof Premier. 


\section{Note on use of drift times versus corrected drift times.}

The apparent resolving powers in RF-confining drift cells are corrected by subtracting $t_{0}$ from $t_{D} . t_{0}$ in those experiments consists of the transit time between the end of the RF-confining drift cell and the time-of-flight mass analyzer, which is predominantly mobility independent ${ }^{2}$ and has values that range between 0.6 and $1.5 \mathrm{~ms}$ for the analytes investigated here. The $t_{D}$ values used to determine the apparent resolving power values for SLIM experiments were not corrected for $t_{0}$ because ion transport outside of the SLIM region is predominantly mobility dependent and contributes to the measured peak widths. The mobility-independent portions of $t_{0}$ in the SLIM experiments is expected to be similar in magnitude to those in the RF-confining drift cell experiments, and thus are very small relative to $t_{\mathrm{D}}$ in the SLIM experiments. 
Table S1. Effective temperatures estimated for the 16+ charge state of native-like avidin in a second-generation RF-confining drift cell (RFG2) or SLIM at the five drift field strengths $\left(E P^{-1}\right)$ used for the SLIM measurements in this study. The corresponding device, RF peak-to-peak amplitude $\left(V_{P P}\right)$, and pressure $(P)$ for each set of trajectories is shown above. Errors represent a $95 \%$ confidence interval.

\begin{tabular}{lccc}
\hline Device & RFG2 & RFG2 & SLIM \\
$V_{P P} / \mathrm{V}$ & 0 & 100 & 200 \\
$P /$ Torr & 1.5 & 1.5 & 4 \\
\hline \multicolumn{4}{c}{ Effective Temperature $/ \mathrm{K}$} \\
\hline$E P^{-1} / \mathrm{V} \mathrm{cm}^{-1} \mathrm{Torr}^{-1}$ & \multicolumn{3}{c}{$298.9 \pm 0.8$} \\
\hline 1.29 & $298.2 \pm 0.4$ & $298.4 \pm 0.8$ & $298.6 \pm 0.7$ \\
1.42 & $298.2 \pm 0.6$ & $297.9 \pm 0.5$ & $298.6 \pm 0.6$ \\
1.60 & $298.0 \pm 0.7$ & $298.5 \pm 0.2$ & $298.9 \pm 0.6$ \\
1.82 & $298.3 \pm 0.6$ & $298.1 \pm 0.6$ & $299.3 \pm 0.6$ \\
2.11 & $298.3 \pm 0.6$ & $298.6 \pm 0.5$ & $298.6 \pm 0.7$ \\
\hline
\end{tabular}



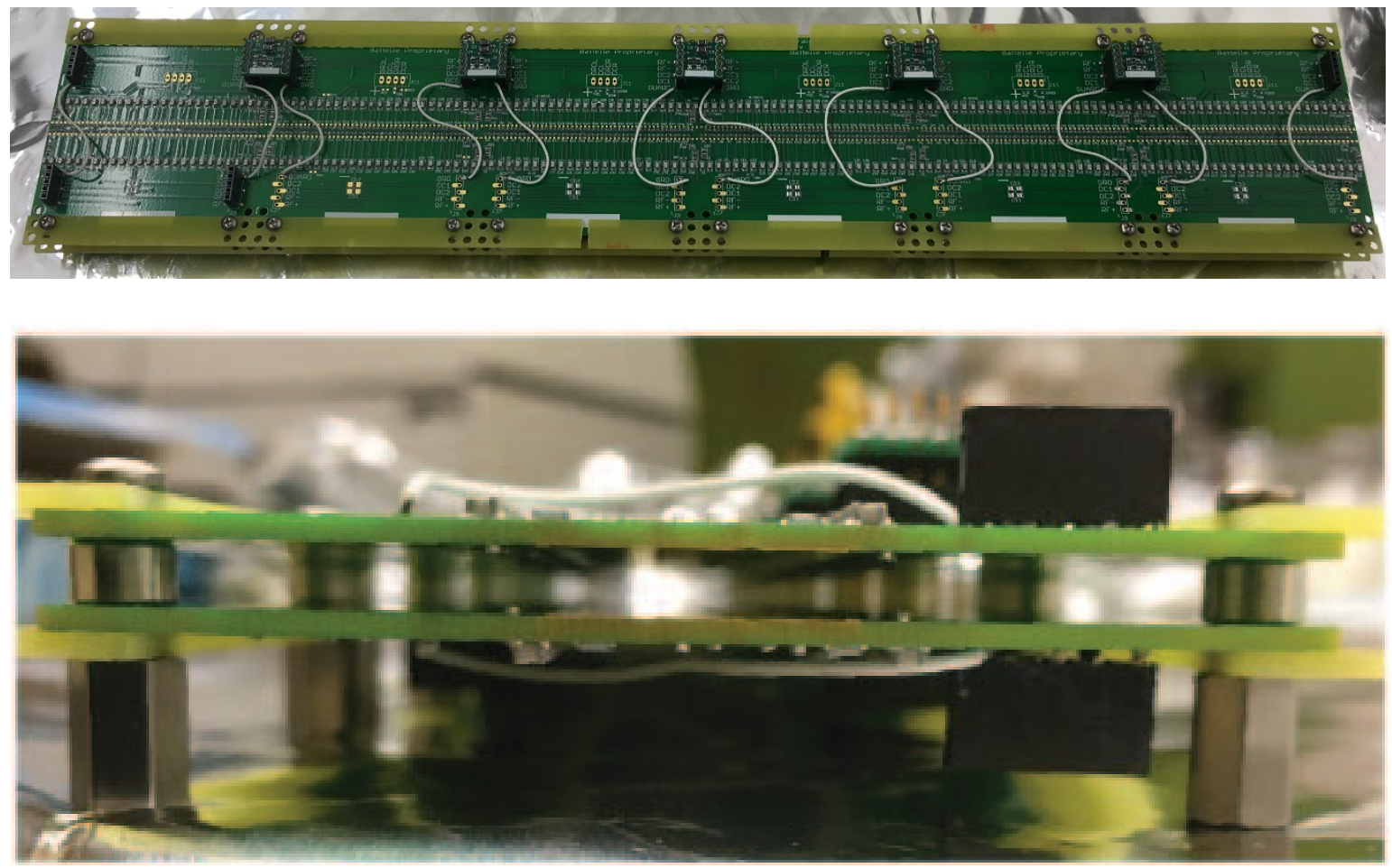

Figure S1. (Top) Picture of SLIM region that contains a total of 12 SLIM boards that are stacked into a linear series of six SLIM board pairs. Top-bottom and neighboring boards are electrically connected using bridging boards. (Bottom) SLIM board are separated by $3.97 \mathrm{~mm}$ length stainless steel spacers. 


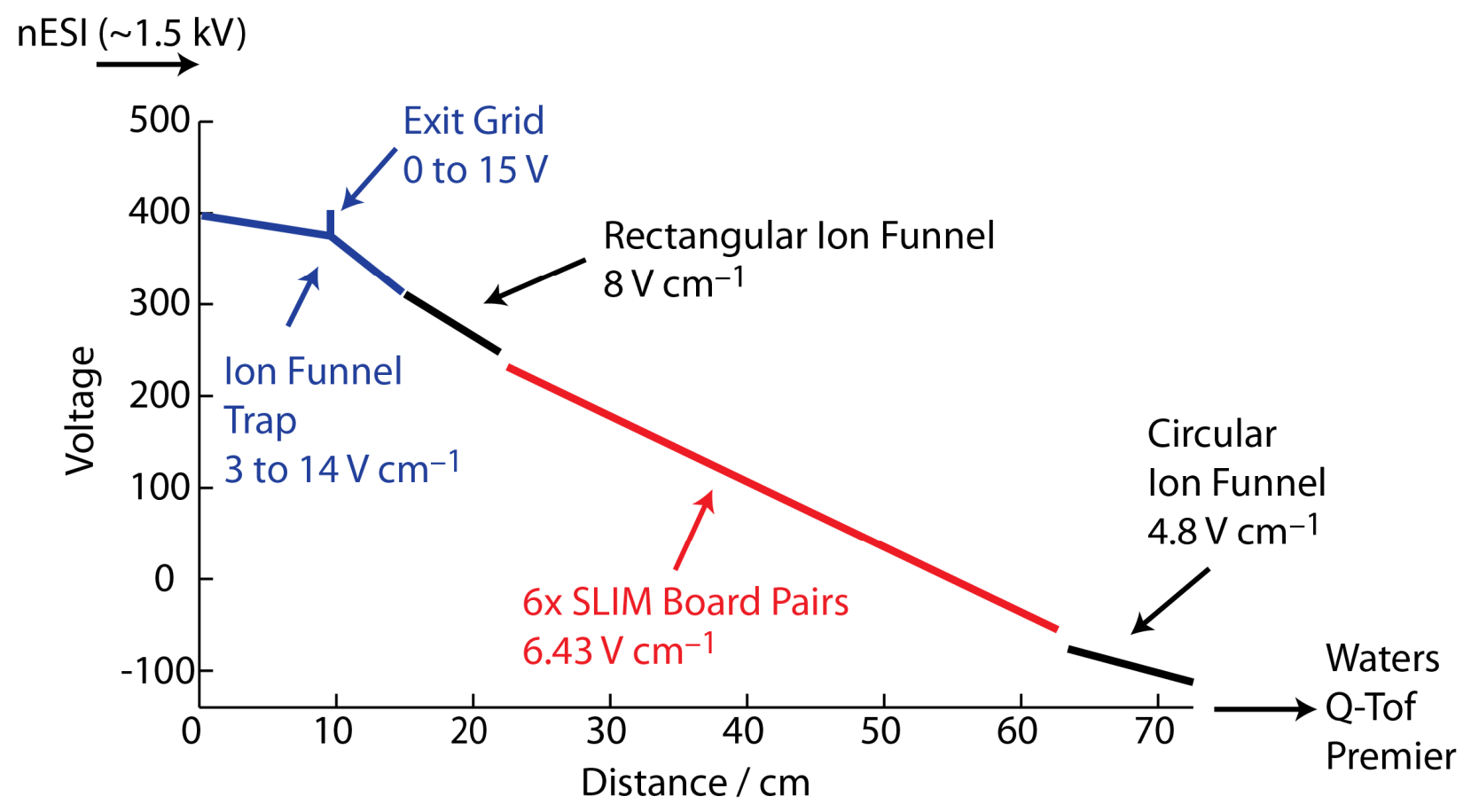

Figure S2. Potential diagram of the new SLIM instrument, including representative values for native IMMS experiments. 


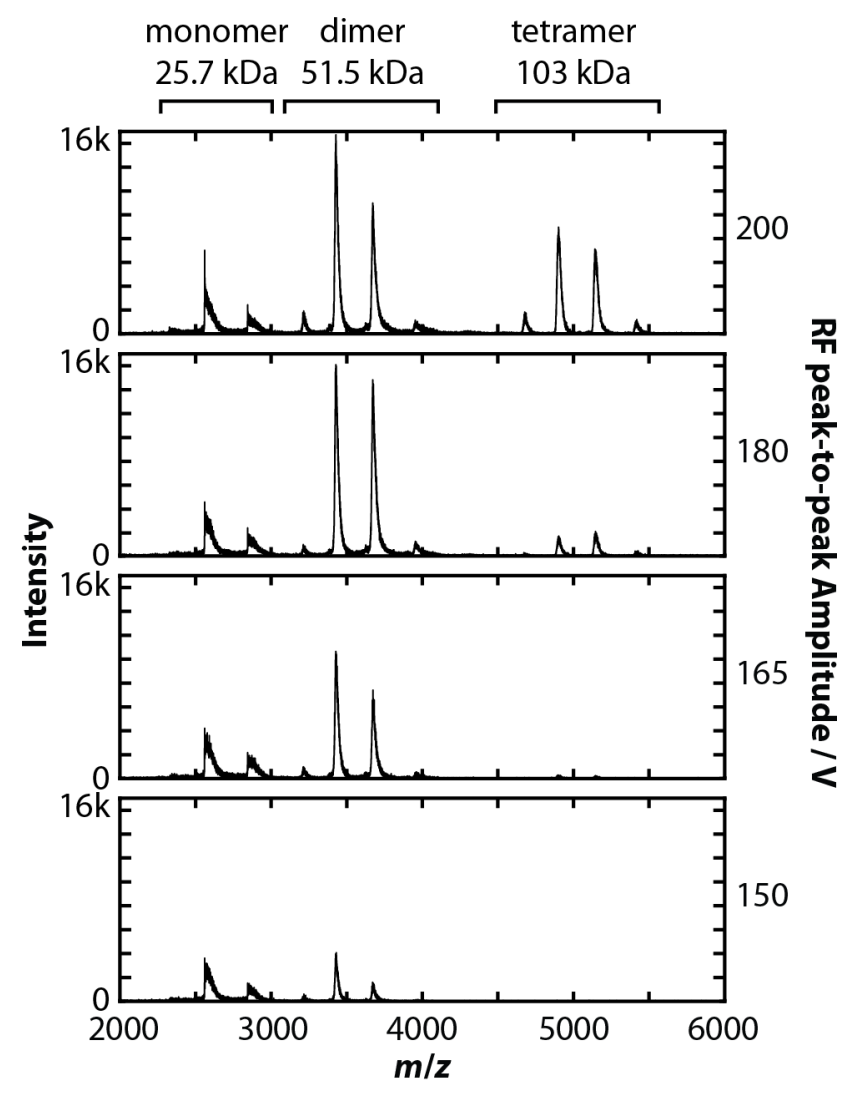

Figure S3. Mass spectra of native-like concanavalin A for SLIM-region RF peak-to-peak amplitudes $\left(V_{P P}\right)$ ranging from 150 to $200 \mathrm{~V}$. As $V_{P P}$ is reduced, higher $m / z$ transmission through the SLIM region decreases. 

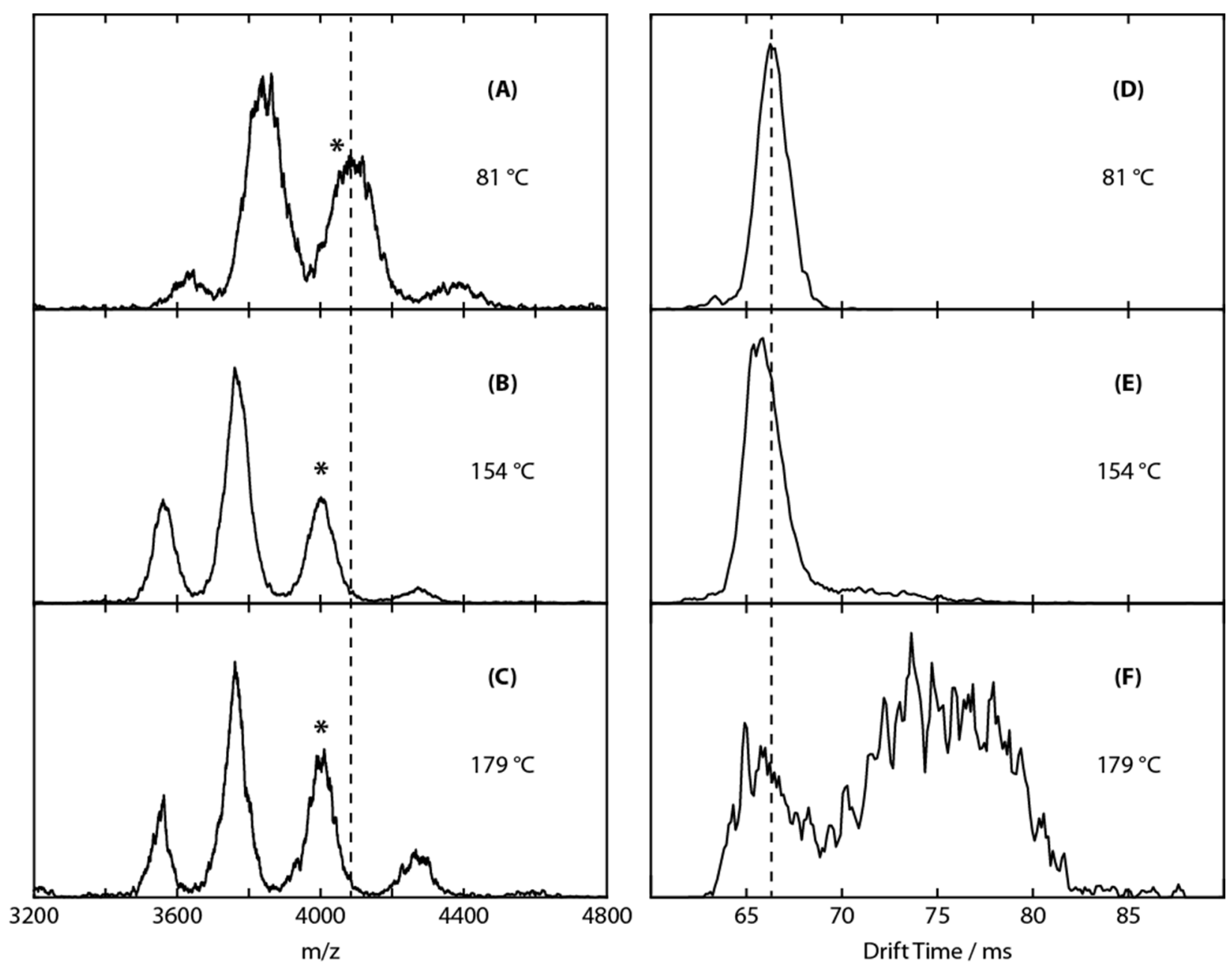

Figure S4. Mass spectra of the native-like, homotetramer avidin (A to C) and arrival-time distributions of $16+$ charge state (D to F) measured at stainless steel inlet capillary temperatures of $81{ }^{\circ} \mathrm{C}\left(\mathrm{A}\right.$ and D), $154{ }^{\circ} \mathrm{C}(\mathrm{B}$ and $\mathrm{E})$ and $179{ }^{\circ} \mathrm{C}(\mathrm{C}$ and $\mathrm{F})$ at a SLIM drift field of $5.73 \mathrm{~V} \mathrm{~cm}^{-1}$. Asterisks mark the $16+$ charge state in the mass spectra (A to C), which was used to monitor protein complex activation. The dotted lines denote either the $m / z$ value or drift time seen when the instrument is operated under non-activating conditions $\left(81^{\circ} \mathrm{C}\right)$. The avidin tetramer ions are activated with increasing inlet capillary temperatures, resulting in the evaporation of residual noncovalent adducts from solution and a reduction in the $\mathrm{m} / \mathrm{z}$ value measured for the $16+$ charge state. Correspondingly, increased activation also results in compaction (E) and then expansion (F) of avidin from its native-like conformation, as is evident from longer drift times. At an inlet temperature of $179^{\circ} \mathrm{C}$, intense features for the monomer were also observed, consistent with the dissociation and decreased intensity of the tetramer. An inlet capillary temperature $\sim 80{ }^{\circ} \mathrm{C}$ was found to aid in droplet desolvation while maintaining a native-like structure of avidin. 


\section{References}

(1) Chen, T.-C.; Webb, I. K.; Prost, S. A.; Harrer, M. B.; Norheim, R. V.; Tang, K.; Ibrahim, Y. M.; Smith, R. D. Analytical Chemistry 2015, 87, 716-722.

(2) Ruotolo, B. T.; Benesch, J. L. P.; Sandercock, A. M.; Hyung, S.-J.; Robinson, C. V. Nat. Protoc. 2008, 3, 1139-1152. 